\title{
WISH-R- a fast and efficient tool for construction of epistatic networks for complex traits and diseases
}

\author{
Victor A. O. Carmelo ${ }^{1,2}$, Lisette J. A. Kogelmann ${ }^{2,3}$, Majbritt Busk Madsen ${ }^{4}$ and Haja N. Kadarmideen ${ }^{1,2^{*}}$ (D)
}

\begin{abstract}
Background: Genetic epistasis is an often-overlooked area in the study of the genomics of complex traits. Genome-wide association studies are a useful tool for revealing potential causal genetic variants, but in this context, epistasis is generally ignored. Data complexity and interpretation issues make it difficult to process and interpret epistasis. As the number of interaction grows exponentially with the number of variants, computational limitation is a bottleneck. Gene Network based strategies have been successful in integrating biological data and identifying relevant hub genes and pathways related to complex traits. In this study, epistatic interactions and network-based analysis are combined in the Weighted Interaction SNP hub (WISH) method and implemented in an efficient and easy to use R package.

Results: The WISH R package (WISH-R) was developed to calculate epistatic interactions on a genome-wide level based on genomic data. It is easy to use and install, and works on regular genomic data. The package filters data based on linkage disequilibrium and calculates epistatic interaction coefficients between SNP pairs based on a parallelized efficient linear model and generalized linear model implementations. Normalized epistatic coefficients are analyzed in a network framework, alleviating multiple testing issues and integrating biological signal to identify modules and pathways related to complex traits. Functions for visualizing results and testing runtimes are also provided.

Conclusion: The WISH-R package is an efficient implementation for analyzing genome-wide epistasis for complex diseases and traits. It includes methods and strategies for analyzing epistasis from initial data filtering until final data interpretation. WISH offers a new way to analyze genomic data by combining epistasis and network based analysis in one method and provides options for visualizations. This alleviates many of the existing hurdles in the analysis of genomic interactions.
\end{abstract}

Keywords: Epistasis, Networks, GWAS, Complex traits, WGCNA

\section{Background}

High throughput genotyping data have been used extensively in many contexts to explain phenotypic variation of complex traits in a wide range of Genome Wide Association Studies (GWAS). GWAS can however, only partially explain observed phenotypic variation [1], and phenotypic variation has been shown to eclipse

\footnotetext{
* Correspondence: hajak@dtu.dk

'Quantitative and Systems Genomics Group, Department of Bio and Health Informatics, Technical University of Denmark, Kemitorvet, Building 208, 2800 Kgs. Lyngby, Denmark

${ }^{2}$ Animal Breeding, Quantitative Genetics and Systems Biology group, Department of Large Animal Sciences, Faculty of Health and Medical Sciences, University of Copenhagen, Frederiksberg, Denmark

Full list of author information is available at the end of the article
}

genotypic variation in the same population [2]. For example, in a large study of inflammatory bowel disease (IBD) only $8.2-13.1 \%$ of the variance in disease liability was explained using GWAS [3]. Several factors can explain the missing heritability of complex traits [4], but one often overlooked aspect is epistasis which can contribute to genetic variation in complex traits. Epistasis can have at least two definitions [5], but here we mean the use of genome-wide multi locus genetic interactions to predict phenotypic variation. Epistasis commonly affects phenotypes [6] and is observed in type 1 and type 2 diabetes [7, 8] and IBD [9] risk loci. Thus, quantification of epistasis can improve our understanding of causal genomic variation. 
Calculation of epistasis is a computational challenge, even on modern computing facilities. To calculate first order epistatic interactions, that is, interaction between pairs of genotypes, of $\mathrm{N}$ loci, it is necessary to do minimum $\sim \frac{N^{2}}{2}$ estimates. In the case of a $700 \mathrm{k}$ SNP array, this leads to and order of $2.5 \times 10^{11}$ computations and a large memory consumption, both generally intractable. Therefore, it is important to have strategies to properly filter and reduce input data dimensionality. In general, when analyzing a specific trait it is assumed that most variants are not causal or associated with the trait. Furthermore, many variants will be in high linkage disequilibrium (LD) when using modern high-density genotyping arrays, meaning that their resulting interactions will be highly correlated. Thus, it is not only necessary to filter the input space due to computational issues, but also meaningful from an analysis perspective.

Beyond computational issues, interpretation of epistatic interactions can also be difficult. As the number of tests increases to the square of the input, multiple testing correction will be very stringent, making it difficult to rely on individual interactions. From a biological perspective, it would be useful to look at groups of genes and pathways instead of focusing on single variants. One way of integrating and combining signal from multiple sources is to use network-based strategies. Using networks-based methods is a useful and successful approach in identifying pathways and genes related to complex traits $[10,11]$. A widely used method for this is the WGCNA method and R package [12]. WGCNA is designed for gene expression data, creating networks of co-expressed genes. To take advantage of this feature in a genomic context, the WISH (Weighted Interaction SNP Hub) method was developed by Kogelman and Kadarmideen [13]. WGCNA is built on the assumption that genes that are co-expressed are functional in similar pathways. WISH extends this hypothesis into the assumption that loci that show epistatis are functionally related. WISH calculates epistasis and creates biological networks based on said interactions. The goal is to identify modules of interacting loci that affect a phenotype or complex trait of interest.

We have developed an efficient and easy to use $\mathrm{R}$ package based on the WISH method and added several features including LD based data dimensionality reduction. Using input genotypes and a phenotype the WISH R package filters the data, calculates genome-wide epistatic interactions and generates biologically meaningful networks.

\section{Implementation}

Inputs and filtering

The WISH R package is based on the WISH method [13]. The input files required for the method are a pedigree (ped) and a transposed ped (tped) file, both following standard PLINK format [14]. The overall workflow is shown in Fig. 1. We highly recommend that the raw phenotype data are adjusted for fixed effects and covariates such as sex, age etc., before running genome-wide epistatic model, as they need to be estimated only once. This is done in simple linear regression model fitting all non-genetic fixed effects and, obtaining estimated effects and correct the phenotypes accordingly. We recommend running a simple GWAS on your data first, and then filtering input SNPs based on significance. This helps reduce data dimensionality, as variants with no main effect are unlikely to have epistatic effects, as these would show up at least partially in the main effect estimation. However, we do not recommend strict filtering, as the efficiency of our implementation allows testing of a large number of

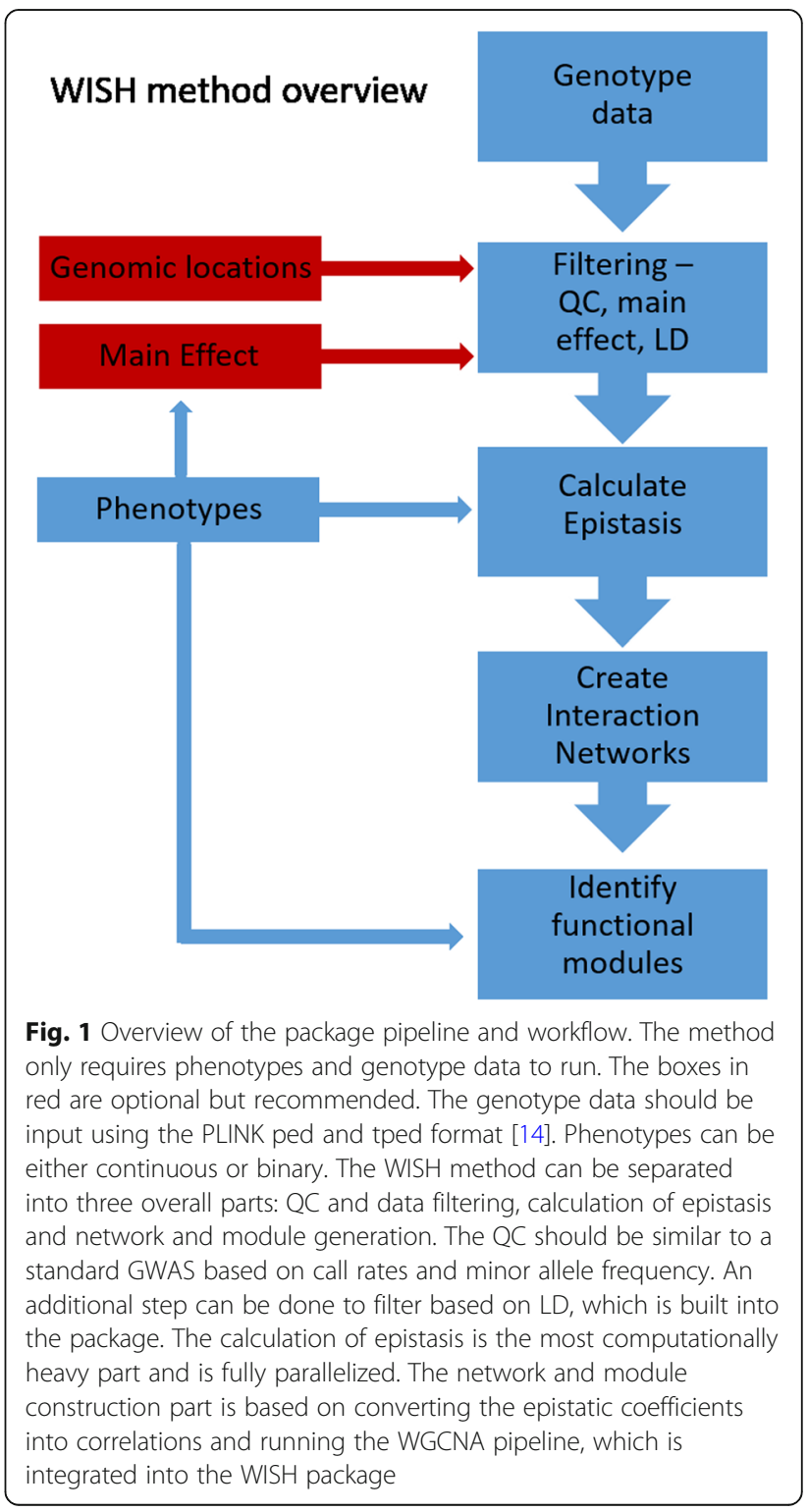


interactions, as discussed further below. This means we recommend including as many variants as feasible depending on the available computational power.

Once a suitable set of variants has been selected it is possible to further filter the data by using LD. Variants in high LD are redundant and will lead to the same nearly identical models being estimated several times. In the context of WISH-R we are not interested in a probabilistic measure of LD, but in the observed LD in a given dataset. If an allele is co-occurring with another allele in a data set they will yield similar epistatic interactions regardless of allele frequencies and sample sizes and we therefore use the $\mathrm{r}^{2}$ measure of LD [15]. In practice, we calculate LD between variants by sliding linearly along the genome, including variants into blocks as long as the mean $r^{2}$ values between all variant pairs is above a selected threshold. When the blocks are identified, the variant with the highest average $r^{2}$ in the block is selected as a representative for the block.

\section{Epistatic interaction modelling}

The main computational challenge is the calculation of epistatic interactions. Therefore, we have several tools to optimize the calculations of the models. The model used for calculating the epistasis is a heterogeneity model $[16,17]$ :

$$
y=\mu+\beta_{1} s n p_{i}+\beta_{2} s n p_{j}+\beta_{3}\left(s n p_{i} \times s n p_{j}\right)+\epsilon
$$

Here $y$ represents a phenotype of interest, $\mu$ is the intercept, $\beta_{1}$ and $\beta_{2}$ are the SNP main effects, $\epsilon$ is a noise term and most importantly $\beta_{3}$ represents the epsitasis of the two loci. To represent the genotypes $s n p_{j}$ and $s n p_{i}$ we code genotype data as 2 (homozygote minor alleles), 1 (hetrozygote) and 0 (homozygote major alleles). The selection of the values for the genotype affects the model hypothesis. Here there is an assumption of multiplicative interaction between minor alleles in the two sites. We also test for the opposite but mathematically identical model by reversing the minor and major homozygote labels in one of the loci. This test is in case the interaction is between minor and major alleles. There is one more parametrization available in the package, which is 2 (homozygote minor alleles), 1.5 (heterozygote) and 1 (homozygote major alleles). This parametrization tests interaction on the gradient of one allele pair set to the other allele pair, which means that all four alleles are involved in the interaction. This is more powerful description but also more difficult to fit as it requires all four alleles to be related to changes in the phenotype for an optimal fit. In the package there is also a generalized linear model (GLM) implemented so that case-control studies (where case-control are coded in binary form as 1-0) can be analyzed. The GLM version is about twice as slow as the non-binary version, as it fits an underlying liability threshold models. The basic linear model uses implementations linked to underlying $\mathrm{C}++$ code, ensuring fast computations of epistatic interactions. The algorithm is fully parallelized. A test setting is included to test runtimes based on input data and the number of threads used.

\section{Network and module creation}

The original idea of WGCNA was based on using correlations in expression data to find interconnected gene. From there it is a natural extension to genomic interactions in networks, by converting the epistatic estimates (the $\beta_{3}$ in the model) to correlations by rescaling them from -1 to 1 . This is done by treating the negative and positive $\beta_{3}$ separately to insure that values close to zero correspond to a correlation of zero. The resulting similarity matrix is then used to calculate the topological overlap measure (TOM) [18]. The next steps follow the workflow of WGCNA: the dissimilarity TOM is used to define modules by creating a gene dendrogram and cutting of branches using a tree-cutting algorithm. Modules are then correlated to the phenotype of interest to detect biologically interesting modules. The functions of WGCNA are integrated in the WISH package for optimization of the workflow. For more details, see Kogleman et al. [13].

\section{Visualization and result assessment}

Visualizing high dimensional data from epistasis in an informative and meaningful way can be a challenge. In the WISH $\mathrm{R}$ package, we have implemented several functions for visualizing and summarizing epistatic interactions. The first method is a pseudo Manhattan plot, based on calculating the sum of -log likelihoods for each variant across all tested interactions. See Fig. 2 for an example. Another measure is a genome wide interaction overview, created by calculating quantile values of significance of interaction between chromosomes, as seen in Fig. 3. While this does not give an accurate representation of individual interactions, it does indicate which chromosomes may be hot spots for interactions for a given phenotype. An example can be seen in Fig. 3. The other option is to visualize epistasis between individual chromosomes. This is done by visualizing the strength of epistasis in all pairwise regions of a user-defined size between selected chromosomes (Additional file 1: Figure S1).

\section{Results and discussion \\ Performance}

When dealing with epistasis it is important to have efficient algorithms. We tested the performance of this part of the package using randomly simulated phenotypes and genotypes. In Fig. 4 we can see the runtime of WISH based on different number of variants and 500 samples using different amount of threads. The test where conducted using AMD Opteron 6380 Processors running at 


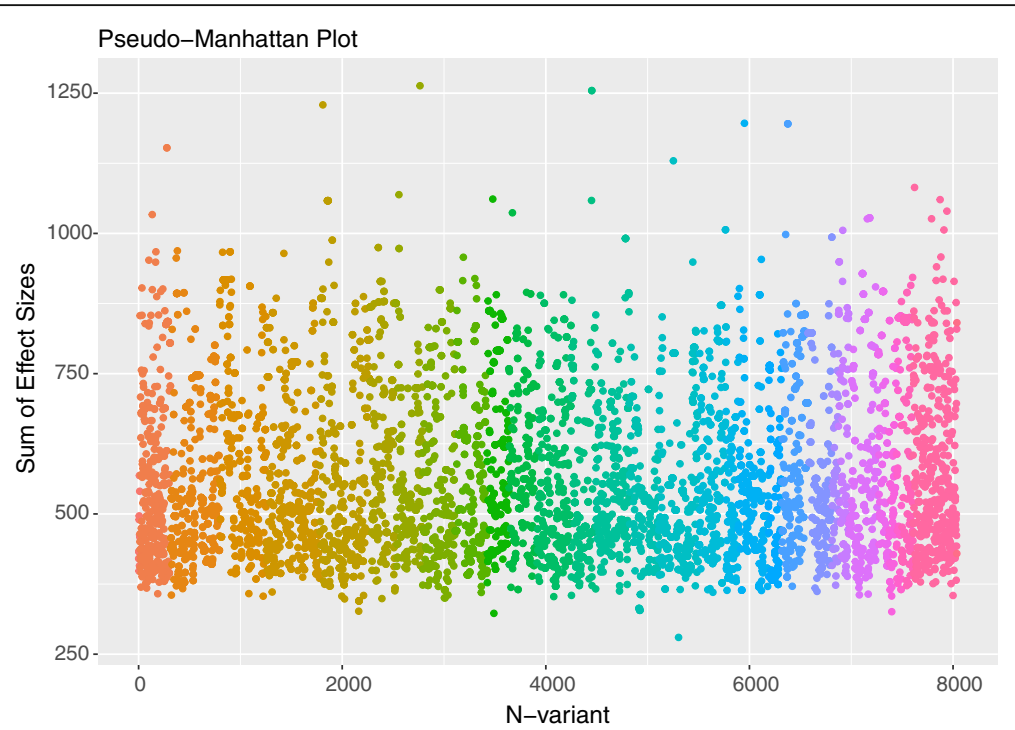

Fig. 2 Example of a Pseudo Manhattan plot. Visualizing interactions in a meaningful way is difficult due to the high data dimensionality. One way to solve this is to use summary statistics for each locus instead. Here we sum over the -log likelihoods of all interactions for each variant to give an idea of which variants are most strongly interacting across the genome and color by chromosome

$2.5 \mathrm{GHz}$ with varying number of cores used. There is an approximately linear increase in run-speed based on the number of threads. With our benchmark, it would take around $3 \mathrm{~h}$ for 10,000 variants or about three days for 50,000 variants using 40 threads. In
Additional file 2: Figure S2 we see that the package is not sensitive to the number of samples, and can therefore be run on a wide range of sample sizes. The LD filtering and network analysis part of the package are entirely dependent on the input data, and do not have any

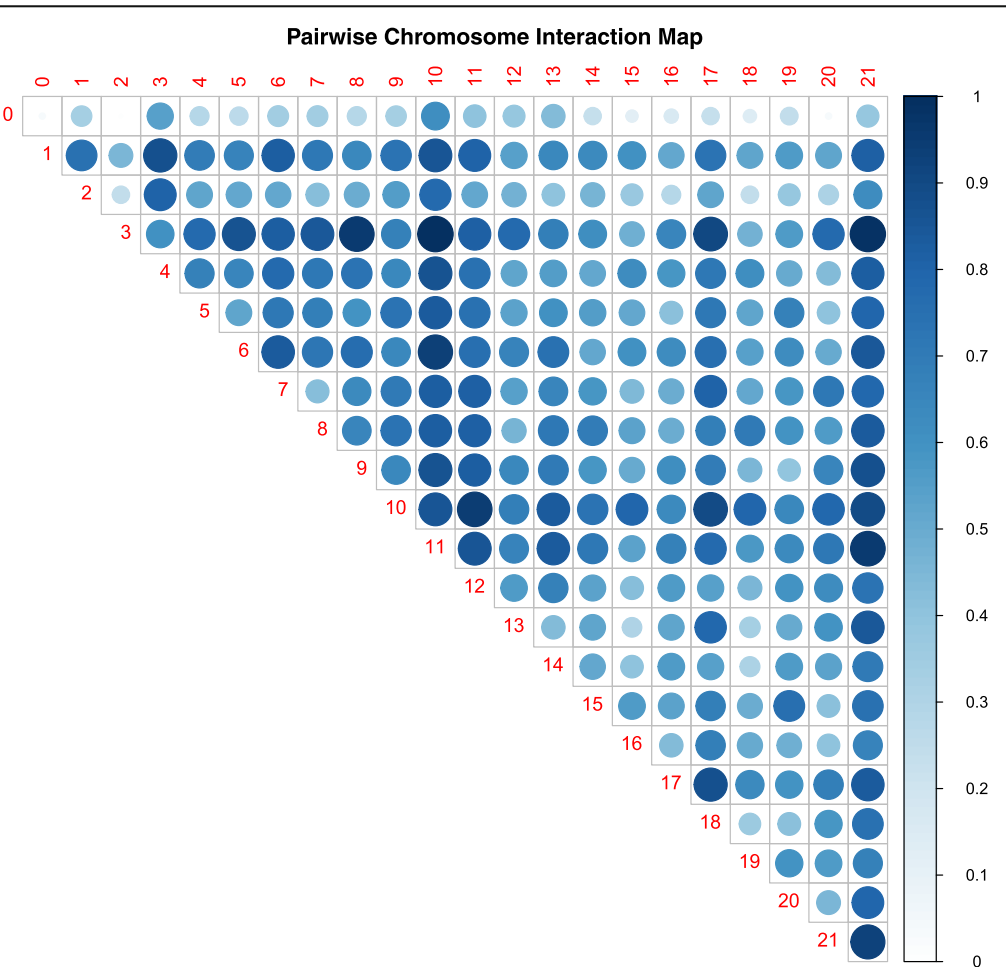

Fig. 3 Visualization of pairwise chromosomal interaction strength. Chromosomal interactions are found by calculating the 90th percentile of the -log likelihood of all epistatic interactions between each chromosome pair and then normalizing them to from - 1 (weakest) to 1 (strongest) interactions 


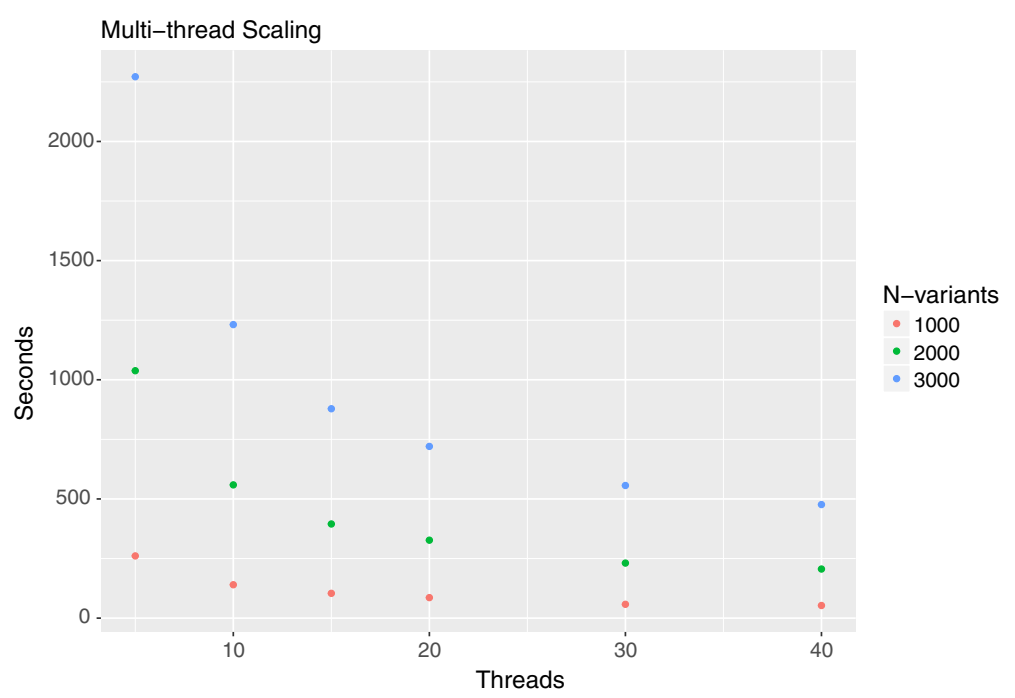

Fig. 4 Scaling of runtime using multithreading based on 1000, 2000 or 3000 variants and 500 samples using simulated genotypes and phenotypes. We see that the improvement in run time with increased number of threads is not linear, due to increased overhead. In all the different runs the improvement in runtime from 5 to 40 threads is ab out 5 -fold. On the other hand, the number of variants has no effect on the speed with about 9000 models per second being calculated using 40 threads across all runs. This is because as with larger data sets the individual threads handle larger data chunks at a time, leading to less overhead

computational challenges. For an example of a full analysis see the original WISH paper $[13,19]$.

\section{Method comparison}

In general, it is difficult to compare methods that calculate epistasis as different models and definitions of epistasis are used. SNPassoc [20] can calculate epistatic interactions but lacks any strategies or recommendations for the computational issues. EPIBLASTER [21] reports being able to calculate a high amount of interactions but requires a GPU computing facility and specific sub-setting and partitioning of the data. Their strategy to filter the data is to a priori calculate simple correlations between cases and controls and variants, as their method only applies to binary phenotypes. This is similar to our suggested approach of using a main effect filtering, however, they end up calculating much fewer interaction models. They report being able to analyze $300 \mathrm{k}$ markers in one day but, using real data they only calculate actual epistasis in 373,153 SNP pairs out of $4.5 \times 10^{10}$ possible pairs. Their implementation does not include the epistasis modelling, requiring more work to get the epistasis results., FastEpistasis [22] has a similar idea as our method for the epistasis calculations, but it only has focus on one aspect, namely calculating the models. They do not discuss filtering strategies or data analysis strategies but are able to calculate the models faster. Martinéz et al. [23] also focus only on epistasis without filtering steps, but report having a higher sensitivity than other available methods, but they do not present any evidence as to why this should be the case. Their implementation has comparable speed to ours.
Boost [24] offers very high performance based on using approximated calculations setups, but is not straightforward to use, as it requires non-standard input files and requires specific GPU computing software and hardware setups. Similar to Boost but more recently, Gonzalez-domingues et al. [25] are able to calculate epistasis for large datasets, but they also use specialized hardware setups and it is unclear if their implementation is generally available. We believe that WISH offers several advantages compared to other models. Our method works both on quantitative and binary phenotypes, and we apply the full model to all pairs in the input space. Most of the above methods are able to calculate epistatic interactions at a faster speed than our implementation, but this comes at a cost. Either heuristic filters are applied, or specific hardware is needed, and often the methods themselves are not straightforward to use. In regards to speed, it is unlikely that it is necessary to calculate epistasis for all SNP pairs on a high-density SNP chip, as many of these calculations will be redundant or not biologically related to the trait of interest. The epistasis calculations of WISH should be fast enough to cover most or all biologically relevant SNPs We present strategies for filtering the data using SNP main effect and we include a built-in LD filter, thus ensuring a proper selection of biologically meaningful SNPs. We also implement a solution for dealing with the epistatic coefficients, namely the application of network-based analysis. Epistasis is in general a very complex subject, and the estimation the epistasis itself is just the start of the analysis. Network analysis is the natural extension of pairwise epistasis, as allows us to identify and analyze more complex 
genomic interaction patterns. One more feature we have that we found lacking in other methods is visualization. Visualizing high dimension epistasis data is technically difficult, but we have included some options for summarized assessment of the epistatic modelling, which we found to be lacking in other methods. Our package is simple to use and implemented in R, making it easy to install, transparent to use, and the outputs are easy to manipulate for the user.

\section{Conclusions}

Epistasis in an important component of genetic variation and may have causal effects in certain diseases or complex trait manifestation in humans, animals, plants and other organisms. However, analysis of epistasis on genome-wide scale is an overlooked subject with several challenges, mainly interpretation and data dimensionality issues. We have previously proposed the WISH method for calculating epistasis and applying the results in a network framework, thus offering solutions for some of the main issues in the analysis of epistasis. Here we have implemented WISH-R, an efficient $\mathrm{R}$ package for calculating linear interaction between genomic variants from standard genotype data and generating modules of groups of interacting variants. WISH-R is easy to install and use, and provides tools for analyzing epistasis in complex traits and diseases based on whole genomic data from data filtering to final interpretation.

\section{Availability and requirements \\ Project name: WISH-R package. \\ Project homepage: https://github.com/QSG-group/ wish \\ Operating system: Platform Independent. \\ Programming Language: $\mathrm{R}$. \\ Other requirements: $\mathrm{R} 3.0$ or $>$. \\ License: GPL-3. \\ Restrictions to use by non-academics: license needed.}

\section{Additional files}

Additional File 1: Figure S1. Example visualization of the package function pairwise.chr.map() function displaying the strength of epistatic interaction between regions on two chromosomes. (DOCX $38 \mathrm{~kb}$ )

Additional File 2: Figure S2. Visualization of the runtime scaling of the method based on changes in sample size. (DOCX $27 \mathrm{~kb}$ )

\section{Abbreviations}

GLM: Generalized linear model; GWAS: Genome Wide Association Studies; IBD: inflammatory bowel disease; LD: Linkage disequilibrium; Ped: pedigree file; TOM: Topological overlap measure; Tped: transposed ped file; WISH: Weighted Interaction SNP hub; WISH-R: The WISH R package

\section{Funding}

VAOC was supported by FeedOMICS project which is funded by a grant from the Danish Council for Independent Research - Technology and Production (DFF-FTP Grant Number 4184-00268) and the Danish Technical University. HNK was the grant holder and Principal Investigator of the
FeedOMICS project. LJAK was supported by a grant from the Candys Foundation. MBM was funded by the research fund of the mental health services, Capital region of Denmark.

Availability of data and materials

WISH-R package is freely available at https://github.com/QSG-group/wish.

Authors' contributions

VAOC implemented the method and developed the package including optimization of implementation and design and creation of visualizing and filtering methods. HNK and LJAK created and developed the methodology and gave continuous feedback on package development. MBM tested and gave feedback to improve the method, implementation and design of the package. All authors drafted, read and approved the final manuscript.

Ethics approval and consent to participate

Not applicable

Consent for publication

Not applicable

Competing interests

The authors declare that they have no competing interests.

\section{Publisher's Note}

Springer Nature remains neutral with regard to jurisdictional claims in published maps and institutional affiliations.

\section{Author details}

'Quantitative and Systems Genomics Group, Department of Bio and Health Informatics, Technical University of Denmark, Kemitorvet, Building 208, 2800 Kgs. Lyngby, Denmark. ${ }^{2}$ Animal Breeding, Quantitative Genetics and Systems Biology group, Department of Large Animal Sciences, Faculty of Health and Medical Sciences, University of Copenhagen, Frederiksberg, Denmark.

${ }^{3}$ Danish Headache Center, Department of Neurology, Rigshospitalet Glostrup, Nordre Ringvej 69, 2600 Glostrup, Denmark. ${ }^{4}$ Institute of Biological Psychiatry, Mental Health Centre, Sct. Hans, Roskilde, Capital Region of Denmark,

Denmark.

Received: 6 November 2017 Accepted: 18 July 2018

Published online: 31 July 2018

References

1. Visscher PM, et al. Five years of GWAS discovery. Am J Hum Genet. 2012; 90(1):7-24.

2. Shao H, et al. Genetic architecture of complex traits: large phenotypic effects and pervasive epistasis. Proc Natl Acad Sci U S A. 2008;105(50): 19910-4.

3. Liu JZ, et al. Association analyses identify 38 susceptibility loci for inflammatory bowel disease and highlight shared genetic risk across populations. Nat Genet. 2015;47(9):979-86.

4. Manolio TA, et al. Finding the missing heritability of complex diseases. Nature. 2009;461(7265):747-53.

5. Phillips PC. The language of gene interaction. Genetics. 1998;149(3):1167-71.

6. Mackay TFC. Epistasis and quantitative traits: using model organisms to study gene-gene interactions. Nat Rev Genet. 2014;15(1):22-33.

7. Cordell $\mathrm{HJ}$, et al. Two-locus maximum Lod score analysis of a multifactorial trait: joint consideration of IDDM2 and IDDM4 with IDDM1 in type 1 diabetes. Am J Hum Genet. 1995:57(4):920-34.

8. Cox NJ, et al. Loci on chromosomes 2 (NIDDM1) and 15 interact to increase susceptibility to diabetes in Mexican Americans. Nat Genet. 1999;21(2):213-5.

9. Cho JH, et al. Identification of novel susceptibility loci for inflammatory bowel disease on chromosomes 1p, 3q, and 4q: evidence for epistasis between 1p and IBD1. Proc Natl Acad Sci U S A. 1998:95(13):7502-7.

10. Fuller TF, et al. Weighted gene coexpression network analysis strategies applied to mouse weight. Mamm Genome. 2007;18(6-7):463-72.

11. Rotival M, Petretto E. Leveraging gene co-expression networks to pinpoint the regulation of complex traits and disease, with a focus on cardiovascular traits. Brief Funct Genomics. 2014;13(1):66-78.

12. Langfelder $P$, Horvath S. WGCNA: an R package for weighted correlation network analysis. BMC Bioinformatics. 2008;9:559. 
13. Kogelman LJ, Kadarmideen HN. Weighted Interaction SNP Hub (WISH) network method for building genetic networks for complex diseases and traits using whole genome genotype data. BMC Syst Biol. 2014;8(Suppl 2):S5.

14. Purcell $\mathrm{S}$, et al. PLINK: a tool set for whole-genome association and population-based linkage analyses. Am J Hum Genet. 2007;81(3):559-75.

15. Lewontin RC. The interaction of selection and linkage. I General Considerations; Heterotic Models. Genetics. 1964;49(1):49-67.

16. Neuman RJ, Rice JP. Two-locus models of disease. Genet Epidemiol. 1992; 9(5):347-65.

17. Risch N. Linkage strategies for genetically complex traits. I. Multilocus models. Am J Hum Genet. 1990;46(2):222-8.

18. Zhang B, Horvath S. A general framework for weighted gene co-expression network analysis. Stat Appl Genet Mol Biol. 2005;4:Article17.

19. Madsen $M B$, et al. Systems genetics analysis of pharmacogenomics variation during antidepressant treatment. Pharmacogenomics J. 2018;18(1):144-52.

20. Gonzalez JR, et al. SNPassoc: an R package to perform whole genome association studies. Bioinformatics. 2007;23(5):644-5.

21. Kam-Thong T, et al. EPIBLASTER-fast exhaustive two-locus epistasis detection strategy using graphical processing units. Eur J Hum Genet. 2011;19(4):465-71.

22. Schupbach T, et al. FastEpistasis: a high performance computing solution for quantitative trait epistasis. Bioinformatics. 2010;26(11):1468-9.

23. Martínez H, et al. Accelerating FaST-LMM for epistasis tests. Cham: Springer International Publishing; 2017.

24. Wan $\mathrm{X}$, et al. BOOST: a fast approach to detecting gene-gene interactions in genome-wide case-control studies. Am J Hum Genet. 2010;87(3):325-40.

25. Gonzalez-Dominguez J, et al. Parallelizing epistasis detection in GWAS on FPGA and GPU-accelerated computing systems. leee-Acm Trans Computat Biol Bioinform. 2015;12(5):982-94.

Ready to submit your research? Choose BMC and benefit from:

- fast, convenient online submission

- thorough peer review by experienced researchers in your field

- rapid publication on acceptance

- support for research data, including large and complex data types

- gold Open Access which fosters wider collaboration and increased citations

- maximum visibility for your research: over $100 \mathrm{M}$ website views per year

At $\mathrm{BMC}$, research is always in progress.

Learn more biomedcentral.com/submissions 